\title{
Design and Implementation of Financial Warning System based on Large Data
}

\author{
Guoqiang Wu \\ Huainan Normal University, Anhui Huainan 232000 ,China \\ agaaqe@163.com
}

\begin{abstract}
Keywords: Financial management, large data, data aggregation, predictive modeling, meta-data management.
\end{abstract}

\begin{abstract}
This dissertation is going to bring forward modern development and design of Big Data Integration for financial management system, based on limits of traditional financial management and situation under large numbers of business data accumulation. As per development and requirement of financial management, and leading-edge experience and research of financial budget and cost management combined to realities of actual operation and experience, this dissertation is going to research and promote the creative financial management and decision-making system with Big Data integration, which will carry out the capability of centralizing data, reusing valuable large numbers of business data set, providing excellent data analysis and forecasted decision-making analysis.
\end{abstract}

\section{Introduction}

With the comprehensive development and continuous implementation of the information technology during the past more than 20 years, the medical information management is playing much important role in medical operation architecture currently [1]. However, the current medical information management primarily focuses on operational consequently level, Furthermore, the large volumes of business data accumulated are not well-utilized, even wasted, and the analysis based on that can not be better applied to each business unit reasonably, especially being lack of data analysis and diagnosis for decision-making sector. [2] The complexity of medical industry promotes the requirement of business intelligence. [3] Nowadays, the Thinking of Big Data is playing much important role around the world and going to change the layout of traditional business intelligence, to integrate modern management idea and business intelligence better, to promote management level, to optimize the structure of various industries, to improve working efficiencies and productivities and add values. [4] As the promulgation and implementation of the National Financial management and general accounting regulation, the traditional financial management is getting behind of development of modern financial management and requirements from logistics support, requirement of engineer department.

The financial crisis early-warning system based on accounting and financial information is a system that can control and forecast alerts about financial risks that enterprises probably or will face by setting and observing changes of some sensitive financial indicators. With the accelerated pace of international economic integration, the international and domestic market competition that Chinese companies has to face has been becoming increasingly fierce. In order to survive and develop, companies need to establish an effective financial crisis early-warning system to achieve the maximum risk reduction. The financial crisis early-warning system can help enterprises avoid or reduce damages by real time tracking and monitoring the financial position of the enterprises, timely making financial early-warning analysis and early detecting financial distress signs.

\section{Requirement analysis}

According to American scholars Kumpe and Plet analysis, a few decades, the mainstream enterprise patterns have developed from the initial efficiencial oriented enterprise, quality oriented companies, and later flexible enterprise, is now changing to innovative enterprises. Construction o 
innovative companies can provide strong support with building an innovative country, and innovative business-building process is nonlinear complex process with a wide range of innovative cluster types and a number of innovative dynam is integration systems, much more complex risk factors than simply technological innovation process. So it has prominent features of high-risk and high yield. [5]

The financial data and related data are derived from existing medical applications which will import and export financial data to downstream system as per predefined data standard and format declared by China Financial Data Organization for standardization, normalization and customization, and then, promote financial management platform to live, which is to meet with requirement of medical management, to build up financial procedure, such as budget management, cost management and performance management etc. On the other hands, it will provide financial statement, financial trend analysis and decision-making information based on financial data golden source. These are all aligned to transformation from traditional to modern financial management. Metadata table data relationships are shown in Fig. 1.

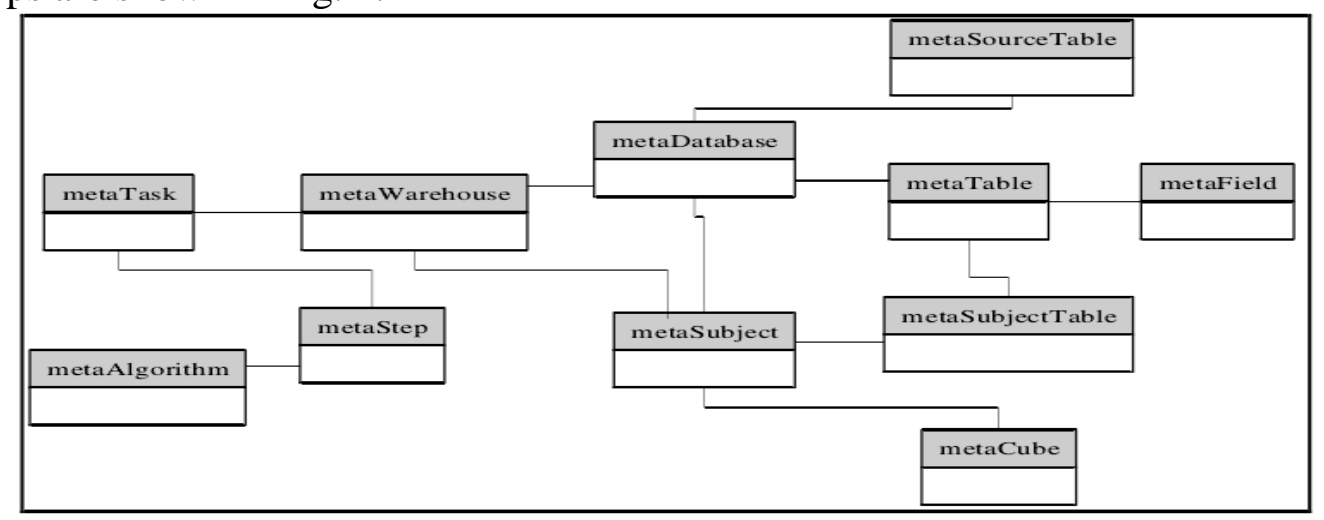

Fig. 1 Metadata table data relationships

\section{Architecture based on SOA}

This application is designed by utilizing leading-edge technology, networking and workflow technology, one of which character is not to impact existing medical application running. Under this model, it is to extract data (structure, semi-structure and unstructured data), refine data and centralize them into unique repository by leveraging standard data model to normalize these data and load them into big-data platform through autopsies job control to schedule batch data processing and real time data processing, through robust ETL and SDM to centralize data into data warehouse with support of meta-data management, [6] data normalization, through matured technology to make sure high-performance data storage, such as database partition, database de-normalization and No SQL technology which is being used as near cache to storage aggregated data in order to ensure application with high-performance and stability. Multi-threaded data query performance test is shown in Fig. 2.
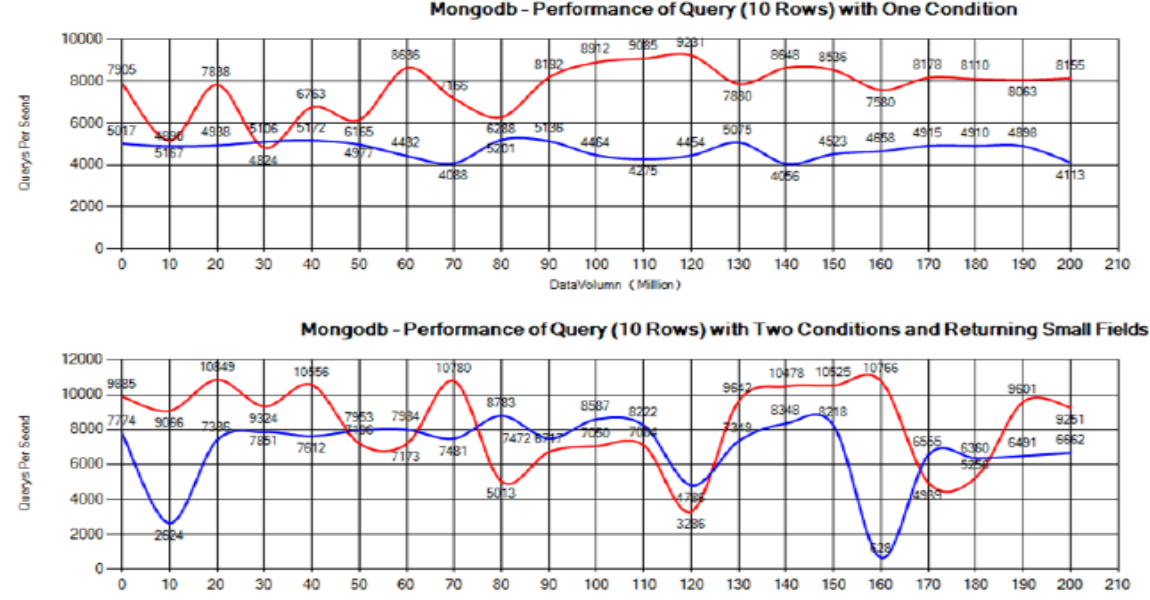

Fig. 2 Multi-threaded data query performance test 


\section{High performance framework}

Server side development is based on J2EE architecture, client side is on Dot Net framework. On server side, it utilize high-performance and document-oriented database MongoDB as background aggregated database, MS SQL server is used as data warehouse. Java and C\# are programming language with Spring framework support and MVC design pattern for rich client development, Hibernate is used as ORM for data persistence. [7] On Client side, WPF is used to build up data visualization layer by leveraging MVVM design pattern. On operation level, Autosys is used for job scheduling to ensure the application $7 * 24$ running. This framework is leveraged Spring framework characteristics to manage business logic object and module whole life cycle with container-helper and non-container helper in order to achieve de-coupling module dependencies internally for high scalability, expansibifity, stability, service-reusability, easy-maintain and fast deployment characteristics. On the other hands, MongoDB is closed to be applied to financial architecture to generate horizontal and vertical dimension data set to store and isolate inventory data, availability data and analyzed data as per different business rule. As MongoDB is near cache implementation, the overall performance is greatly improved to achieve high-effective finance management system.

Iview Service codes are follow as:

using System;

using System.IO;

using System.Collections.Generic;

namespace edu.fmmu.bme.framework.ViewServices.Core

\{public interface IViewService \{

//Registers the given viewName against the given reportType

void Register(viewDefinition viewDefinition);

//Loads the standard view definitions that have been developed

void LoadStandardViewDefinitions(string reportType)

//Loads the view definitions that have been saved

void LoadViewDefinitionsQ;

//Save the given viewDefinition to the user area

void saveViewDefinition(ViewDefinition viewDefinition);

//Get the viewdefinition that was registered against the given parameterref

ViewDefinition GetViewDefinition(Guid id);

//gets a lsist of view names that belong to the given report type

IEumerable $<$ ViewDefinition $>$ GetViewDefinitions(Guid viewTypeId);

$/ /$ Gets a new view name from the given starting name

string GetViewName(Guid viewTypeId, string startName)\}\}

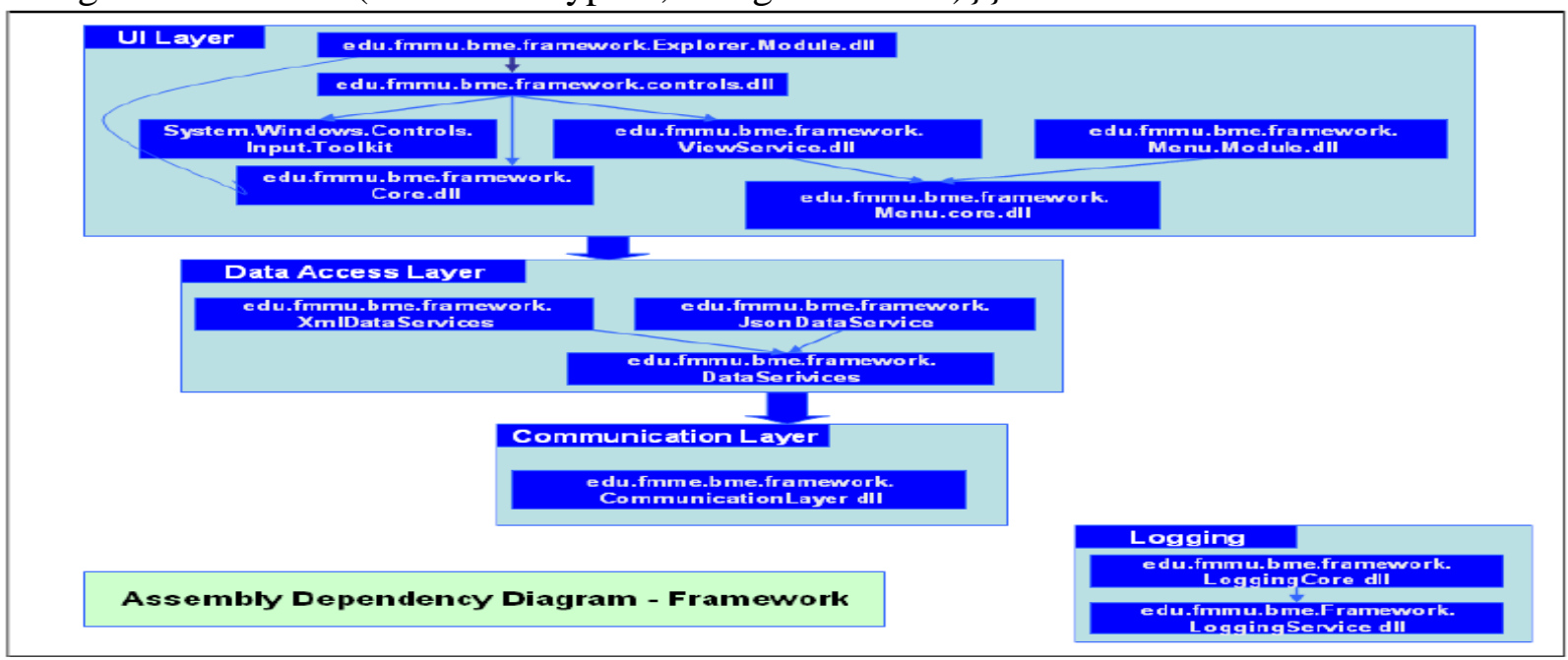

Fig. 3 Dependence schematic

Dependence schematic is shown in Fig. 3. This system is the enhancement of existing medical financial information system, besides the widely-used financial accounting and report, which 
primarily implement real time financial functionality support to satisfy modern financial management requirement and to achieve the goal of medical financial management and decision-making. There are three steps to get Big Data involved into financial system-the storage and management of big data, the management of semi-structured and unstructured data and the analysis of big data about classification, regression, clustering and association rule based on language support in order to cater for simple reporting and advanced predictive modeling as well as deployment. On the other hands, the feedback of this system is better in reality with its outstanding performance advantage based on too much system testing and performance testing.

Finally, the financial risk early-warning information system based on big data was implemented in this paper, which achieves the computerization of the financial risk early warning. The main function of the financial risk early-warning information system includes login module, software management module, importing model data module, model data entry module, and financial early-warning model analysis module. In the design of the software system, the user-friendly graphical interface and the strong degree of integration with early-warning model are achieved based on the consideration of the actual application. In addition, in this paper, the financial risk early-warning information system was verified by comparing the results of manual calculation and software output. The results show that the financial risk early-warning information system can accurately reflect the established early-warning model.

\section{Conclusions}

To summarize the common problem and short-coming from existing medical financial management, to use banking mature financial management experience and idea as reference, this system is built up based in order to improve medical financial management with data analysis and decision-making capability. In the meantime, it keeps the core of data warehouse and business intelligence, integrate thinking of Big Data processing and centralize financial data with standard data format, by which, to promote smart, extendable and robust software architecture, to reinforce financial data interoperability, improve high-performance data aggregation, analysis and dynamic data visualization. On the other hands, it is going to bring in financial Audit and interface of in-depth data mining, which are all added values to proactive significance of medical financial management.

\section{References}

[1] Stefano M. Iacus. Simulation and inference for stochastics differential euqations. Springer, 2008.

[2] Bram Smeets, Seth Ladd. Building spring 2 enterprise applications. New York, NY: Apress, 2012: 67-69.

[3] Information on http://incubator.apache.org/chukw/

[4] Altman E. I. Financial Ratios, Discrirninant Analysis and the Prediction of Corporate Bankruptcy. Journal of Finance, 2011, 9: 589-609.

[5] Fanning K. M., Kogger K. O. A. Comparative Analysis of Artificial Neural Networks using Financial Distress Prediction. International Journal of Intelligent System vi Accounting Finance and Management, 2014, 3(4): 241-252.

[6] Atiya A. F. Bankruptcy Prediction for Credit Risk Using NeuralNetworks: A Survey and New Results. IEEE Transactions on Neural Networks, 2001, 12(4): 929-935.

[7] Charalambous Chris, Andreas Charitou, Froso Kaourou. Comparative Analysis of Artificial Neural Network Models: Application in Bankruptcy Prediction. IEEE Proceedings, 2009, 139(3): 23-31. 